\title{
DINÁMICA Y MORFOLOGÍA GLACIAR EN EL VALLE DE CARDAÑO, PALENCIA (CORDILLERA CANTÁBRICA)
}

\author{
José María Redondo Vega \\ Javier Santos González \\ Departamento de Geografía y Geología. Universidad de León \\ jmredv@unileon.es, jsango@unileon.es
}

\section{RESUMEN}

Se presentan nuevos datos acerca de la importancia de la glaciación cuaternaria en el valle de Cardaño, en el norte de la provincia de Palencia. A partir del método AABR (Area Altitude Balance Ratios) se ha estimado que la altitud de la línea de equilibrio glaciar (LEG) durante el máximo avance glaciar se situó a 1840 m. A pesar de ese elevado valor, la gran superficie existente por encima de esa cota permitió que se desarrollara un glaciar de gran entidad, del que se conservan numerosas formas erosivas y deposicionales, muchas de ellas no observadas anteriormente.

Palabras clave: Glaciarismo; Altitud de la línea de equilibrio glaciar; Método AABR; Cordillera Cantábrica; Patrimonio Geológico.

\section{ABSTRACT}

New data about the glacial geomorphology of Cardaño valley (North of Palencia province, Spain) are presented. Using AABR (Area Altitude Balance Ratios) method we have estimated the ELA position in the last maximum advance at $1840 \mathrm{~m}$. Instead of this high altitude, the great surface above this altitude permitted the development of an important glacier. Numerous erosional and depositional forms are widespread in the valley, and many of them are recognized for the first time.

Fecha de recepción: septiembre 2011.

Fecha de aceptación: enero 2013. 
Key words: Glacial geomorphology; Equilibrium Line Altitude; AABR method; Cantabrian Mountains; Geomorphosites.

\section{INTRODUCCIÓN}

Durante al menos la última fase glaciar del Cuaternario, las condiciones de temperatura y precipitación fueron suficientes para la formación de numerosos glaciares que ocuparon la mayor parte de las cabeceras de los valles de la Cordillera Cantábrica. Su pasada existencia queda probada por la gran cantidad de formas glaciares que podemos encontrar desde la Sierra de Ancares (entre Lugo y León) al macizo de Castro Valnera (entre Burgos y Cantabria), destacando los circos, los valles en forma de $\mathrm{U}$ o las cubetas, en algunos casos ocupadas por lagunas. Más escasas son las formas de acumulación, las morrenas, que solo en algunos

Figura 1

LOCALIZACIÓN DEL ÁREA DE ESTUDIO

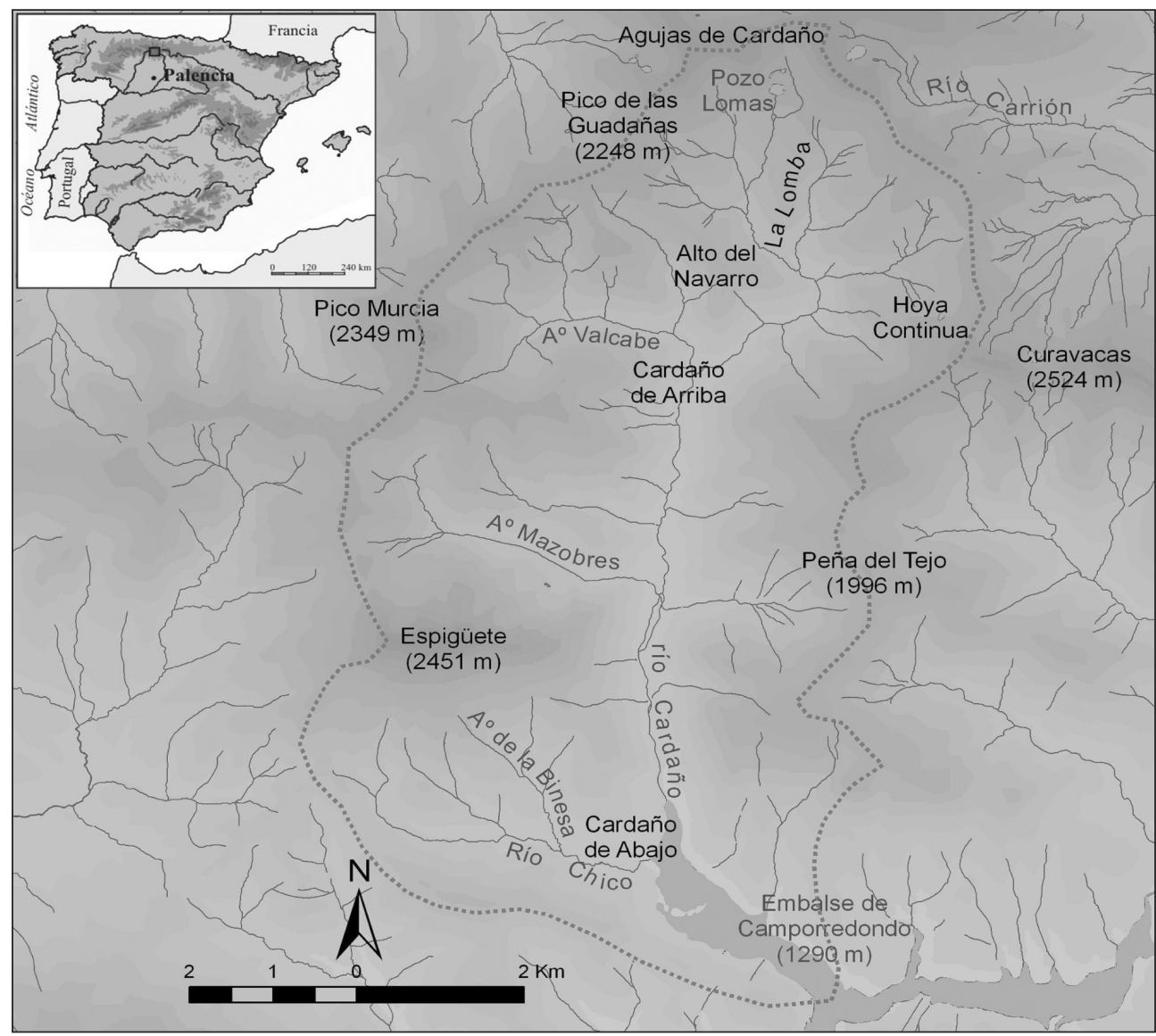


valles aparecen bien definidas, ya que frecuentemente han sufrido una intensa erosión postglaciar, que ha sido más notable en las vertientes de los ríos cantábricos y de la cuenca del Miño.

A pesar de la importancia de la glaciación en el relieve de buena parte de la Cordillera, los estudios sobre la dinámica glaciar han sido relativamente escasos y solo recientemente se ha demostrado la gran magnitud que alcanzaron las lenguas de hielo en algunos de los valles (Pérez Alberti y Valcárcel Díaz, 1998; García de Celis y Martínez Fernández, 2002; González Gutiérrez, 2002; Redondo Vega, 2002; Alonso y Suárez Rodríguez, 2004; González Trueba, 2006; Jalut et al., 2010; Santos González, 2012).

El valle de Cardaño, situado en el norte de la provincia de Palencia (Fig. 1), reúne una serie de condiciones morfoestructurales, litológicas y altitudinales que hacen que sea uno de los de mayor interés para el estudio del glaciarismo cuaternario de la Cordillera Cantábrica.

\section{ANTECEDENTES}

La primera referencia sobre el modelado glaciar en la zona, en el cercano valle de Lechada, es de mediados del s. XIX, en relación con la presencia de bloques erráticos de granito en la zona del Boquerón de Bobias (Prado, 1852), si bien este autor, en ese momento, no considera claramente un transporte glaciar. En el mismo trabajo describe un pequeño glaciar al norte de Peña Prieta, en el Cubil del Can (enclave que, incluso en la actualidad, suele mantener nieve durante casi todo el año), en la única referencia clara realizada a una masa de hielo durante la Pequeña Edad del Hielo en la Cordillera Cantábrica fuera de Picos de Europa (Prado, 1852: 172). A finales del siglo XIX, Saint Saud (1893) indica la presencia de nieve perpetua (quizás un helero) en la vertiente septentrional del Espigüete, donde durante algunos siglos se recogía nieve para comerciar con otras zonas de la meseta (Gutiérrez Álvarez, 2007) y dónde casi todos los años conserva algún nevero a finales de verano.

Más adelante, Nussbaum y Gigax (1953) citan la presencia de una morrena a la altura de Cardaño de Abajo, que indicaría la máxima posición que alcanzó el hielo. Algo más recientes son las descripciones generales sobre los restos de origen glaciar de esa parte de la Montaña Palentina realizadas por Savage (1967), o la primera cartografía que recoge la mencionada morrena de Cardaño de Abajo, que figura en la hoja del Magna (Rodríguez Fernández, 1984).

Años después, Alonso Herrero (1987) realiza un estudio de la glaciación en la cabecera del sistema fluvial del Esla y, aunque se ajusta a la vertiente leonesa del cordal Pico Murcia-Peña Prieta (incluye no obstante, diagramas de orientación de los circos en ambas vertientes), se pone de manifiesto la magnitud e importancia que tuvieron para el autor las glaciaciones cuaternarias.

La primera descripción minuciosa del relieve glaciar del valle de Cardaño la realiza Castañón Álvarez (1989) en su tesis doctoral. Para este autor aquí se formó una de las lenguas de mayor tamaño de toda la Cordillera, con $10,8 \mathrm{~km}$ y frente a $1280 \mathrm{~m}$. Se describen las formas glaciares y su relación con las estructuras geológicas y se plantea que el máximo avance del hielo se situó en el arco morrénico mencionado ya anteriormente por otros autores. Similar planteamiento recibe el valle de Cardaño en estudios posteriores en los que se aborda el modelado glaciar del Macizo de Peña Prieta (Frochoso Sánchez y Castañón Álvarez, 1996), y de la Cordillera Cantábrica (Frochoso Sánchez y Castañón Álvarez, 1998); en este último 
trabajo se incluye una detallada cartografía geomorfológica pero centrada en el valle principal del río Carrión, ya que del de Cardaño únicamente se representa la cabecera.

Ya en el siglo actual se lleva a cabo un inventario de Lugares de Interés Geológico (LIG) de la provincia de Palencia (Heredia Carballo y Rodríguez Fernández, 2001), pero vuelven a centrarse en el valle principal del Carrión dejando al margen el de Cardaño. Por nuestra parte, en el año 2008 se efectúa un reconocimiento integral del valle de Cardaño dentro de la realización de un inventario de LIG de Palencia (Fernández Martínez et al., 2009), dando como resultado la aparición de nuevos restos glaciares de gran interés, que permiten reconstruir con mayor detalle los límites y la extensión de la glaciación en este valle.

\section{METODOLOGÍA}

Se ha llevado a cabo un intenso reconocimiento de campo, especialmente de la parte baja del valle, con el fin de definir los límites que alcanzaron los hielos. De especial importancia ha sido el afloramiento de granodioritas presente en la cabecera (entorno de las Agujas de Cardaño), ya que se trata de una roca de gran dureza y de la que se encuentran numerosos fragmentos en las laderas a lo largo de todo el valle, en muchos casos constituyendo bloques erráticos apoyados sobre afloramientos de arenisca o de caliza.

Una vez definidos los límites del glaciar a partir de criterios morfológicos y sedimentarios, se ha realizado una reconstrucción de su hipsometría a partir del uso de un Sistema de Información Geográfica (ArcGis 9.2) con el objetivo de poder determinar la altitud de la línea de equilibrio glaciar (LEG), conocida frecuentemente por sus iniciales en inglés de ELA (Equilibrium Line Altitude). Para ello hemos utilizado el método AABR (Area Altitude Balance Ratios).

Este método se basa, al igual que el denominado AAR (i: Accumulation Area Ratio) (Meier y Post, 1962; Torsnes et al., 1993), en el principio de que un glaciar tiene un porcentaje de superficie fijo por encima de la LEG por lo que, conociendo ese valor y sabiendo la topografía del glaciar, se puede estimar la posición de la línea de equilibrio, que se sitúa en la cota por encima de la cual está ese porcentaje de superficie. En glaciares actuales, ese valor oscila casi siempre entre el $50 \%$ y el $80 \%$, siendo del $65 \%$ el más habitual para latitudes medias.

La mayor debilidad del método AAR es que no tiene en cuenta la hipsometría del glaciar, es decir, la distribución de su superficie en relación con la altitud, lo que puede tener importantes repercusiones en la LEG (Benn y Evans, 1998; Rea, 2009). Por eso, está siendo rápidamente reemplazado por el AABR, que sí tiene en cuenta la hipsometría del glaciar. Este método fue establecido por Furbish y Andrews (1984) y desarrollado después por otros autores (Benn y Gemmell, 1997). Osmaston (2005) crea una hoja de cálculo a través del programa Excel®, que facilita el cálculo de la LEG a partir del método AABR. Por su parte, Rea (2009) calcula los valores AABR de glaciares actuales en distintas zonas del planeta, que son de gran utilidad para su uso en reconstrucciones de paleoglaciares. Para este trabajo hemos utilizado los valores que este autor establece para glaciares actuales de la zona alpina y atlántica de latitudes medias, puesto que consideramos que debieron ser los más parecidos al glaciar del Cardaño, y oscilan entre 0,99 y 2,19 y son los que hemos usado para establecer los umbrales máximo y mínimo de la LEG. 


\section{EL CONTEXTO MORFOESTRUCTURAL}

El valle de Cardaño es un corto y muy pendiente valle afluente del río Carrión que nace en las altas cumbres de la divisoria septentrional de la provincia de Palencia con León (Pico Lomas, $2457 \mathrm{~m}$ ). Sigue una trayectoria N-S para desembocar en el mencionado río por su margen derecha, a una cota aproximada de $1260 \mathrm{~m}$ (hoy bajo el embalse de Camporredondo) tras unos $15 \mathrm{~km}$ de recorrido.

Las pendientes muy fuertes que hay entre la divisoria y el río Carrión han creado un elevado potencial morfogenético, aprovechado por un conjunto de arroyos bien alimentados por abundantes precipitaciones y que dibujan una red casi ortogonal. El colector principal, el río Cardaño, sigue una trayectoria N-S y corta, de forma prácticamente perpendicular, las estructuras paleozoicas (contactos, cabalgamientos) alineadas ONO-ESE. Así, el valle de Cardaño atraviesa de $\mathrm{N}$ a $\mathrm{S}$ en su corto recorrido seis de las unidades y formaciones de esta parte de la Cordillera Cantábrica: la intrusión de granodioritas (Rodríguez Fernández, 1994) en el extremo septentrional, y las de Curavacas, Alto Carrión, Espigüete, Cardaño-Resoba y Sierra del Brezo (Rodríguez Fernández, 1984).

Por otro lado, los valles afluentes secundarios, sobre todo los de la margen derecha, se adaptan toscamente a la estructura. Es el caso del río Chico y del $\mathrm{A}^{\circ}$ Mazobres al $\mathrm{S}$ y $\mathrm{N}$ respectivamente del bloque calcáreo del Espigüete $(2451 \mathrm{~m})$, o del $\mathrm{A}^{\circ}$ Valcabe en la vertiente $\mathrm{N}$ del cordal de Pico Murcia (2349 m).

La configuración morfoestructural del relieve preglaciar de la zona tuvo que arrancar en el Mioceno cuando un conjunto de bloques de rocas muy competentes (Espigüete, Curavacas) se levantan por el $\mathrm{N}$ en relación al corredor más meridional de dirección E-O que forma el actual valle del río Carrión (Heredia Carballo y Rodríguez Fernández, 2001). El rejuego de bloques alpino tuvo que tener un carácter diferencial, como lo demuestra el hecho de que el compacto bloque calcáreo del Espigüete domina más de $450 \mathrm{~m}$ la culminación enrasada de la Peña del Tejo (1996 m) sobre los mismos materiales; entre ambos bloques se abre camino hacia el $\mathrm{S}$ el valle del río Cardaño.

Más al $\mathrm{N}$ el valle aparece dominado por el extremo occidental del también compacto y enérgico bloque del Curavacas $(2524 \mathrm{~m})$. En este caso, se trata de un segmento de ese relieve, formando una banda de conglomerados mucho más estrecha, que atraviesa el valle alto de Cardaño de ESE a ONO desde Hoya Continua (2356 m) hasta el Pico de las Guadañas $(2248 \mathrm{~m})$. Aproximadamente en el centro de esa banda, los mismos conglomerados mencionados culminan a $1924 \mathrm{~m}$ en el Alto del Navarro que constituye un bloque claramente deprimido (hundido de 300 a 350 m respecto a las divisorias) y que es cortado por su flanco oriental por la cabecera del río Cardaño. Esos desniveles y saltos tan enérgicos muestran una tectónica de bloques diferencial, cuyas líneas de debilidad ha aprovechado el río Cardaño para labrar el valle en dirección $\mathrm{S}$ y atravesar con relativa facilidad esos roquedos tan competentes.

El relieve preglaciar labrado por la red fluvial afluente del Carrión en este sector montañoso ha sido aprovechado durante los periodos fríos pleistocenos por la dinámica glaciar que, gracias a la buena orientación de los cordales transversales y a la elevada altitud media, se ha transformado en un área de importante acumulación de nieve para la génesis del hielo glaciar. Como consecuencia de la ocupación por el hielo de este relieve, han quedado mues- 
tras muy evidentes, algunas paradigmáticas, de su presencia, tanto desde el punto de vista erosivo como deposicional.

\section{LAS FORMAS DE ORIGEN GLACIAR}

\section{Los circos y las lagunas de origen glaciar}

Entre las formas erosivas destacan los circos, algunos espectaculares como el circo complejo que se localiza en la vertiente septentrional del Espigüete, donde la erosión glaciar y la disolución de las calizas se han conjugado (Castañón Álvarez, 1989) para excavar profundos huecos enérgicamente dominados por paredes verticales de calizas.

En el extremo septentrional se localiza un circo orientado al S y dominado por la crestería de las granodioritas de las Agujas de Cardaño. En su fondo, dos cubetas de sobre-excavación a $2120 \mathrm{~m}$ la superior y a $2055 \mathrm{~m}$ la inferior, albergan aún lagunas de origen glaciar separadas por umbrales rocosos pulidos por el hielo. Las lagunas superiores (de dos a cuatro según la época del año) ocupan una pequeña cubeta de 0,36 ha, estrecha y alargada, labrada por

Figura 2

CUBETAS DE SOBRE-EXCAVACIÓN CERRADAS POR UMBRALES DE RESISTENCIA QUE ALBERGAN LAS LAGUNAS DE POZO LOMAS VISTAS DESDE LAS AGUJAS DE CARDAÑO. AL FONDO SE OBSERVA EL PERFIL TRANSVERSAL EN ARTESA DEL TRAMO FINAL DEL VALLE DE CARDAÑO Y EL BLOQUE DEL ESPIGÜETE A LA DERECHA DE LA IMAGEN

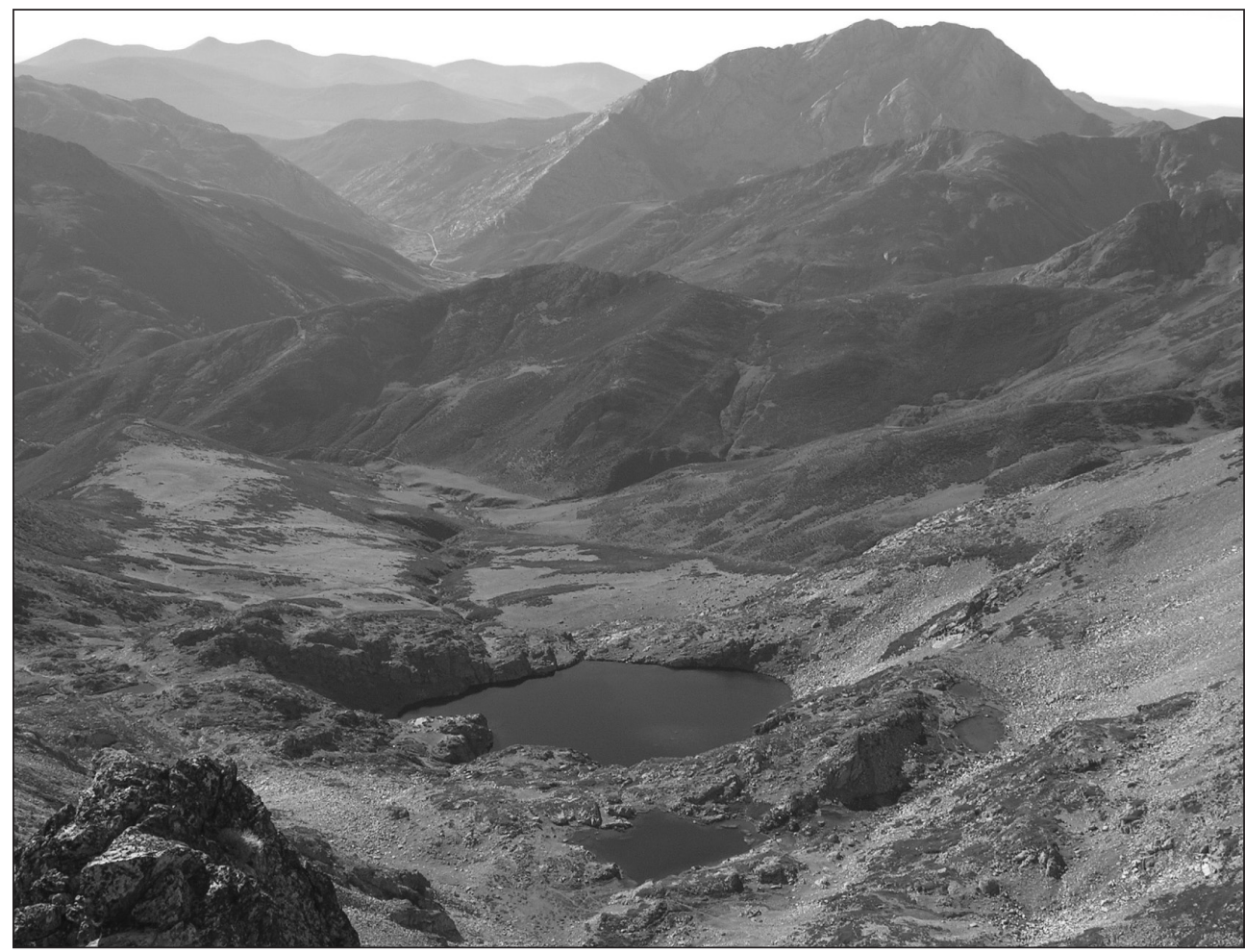


detrás de un umbral de resistencia en las granodioritas; salvo la más oriental, el resto llegan a secarse prácticamente al final del estío.

En la cubeta inferior se localiza el Pozo de Las Lomas (Fig. 2), la laguna de mayor tamaño, con una extensión de 1,98 ha. Se ajusta a una cubeta de sobre-excavación glaciar abierta en el contacto mecánico entre los conglomerados de la Fm. Curavacas, que son los que forman el cerrojo de la cubeta, y las granodioritas que constituyen el circo glaciar que la domina hacia el $\mathrm{N}$.

El circo localizado en el extremo occidental del cordal del Curavacas (pico Hoya Continua, $2356 \mathrm{~m}$ ), está abierto al $\mathrm{N}$ justo en el contacto de los conglomerados con las facies pizarrosas y presenta un umbral con cubeta donde se encuentra la laguna de Hoya Continua. Esta laguna tiene una superficie de 0,45 ha y se sitúa a una cota de $2000 \mathrm{~m}$; en realidad, tiene un carácter estacional pues solamente los años de fuerte innivación tiene agua permanentemente. La laguna está cerrada hacia el $\mathrm{N}$ por un pequeño arco morrénico generado en la fase de circos y que se apoya hacia el $\mathrm{O}$ en un resalte de los conglomerados de la Fm. Curavacas.

Además de estas formas de gran tamaño, aparecen algunas de detalle dentro del valle que son realmente ejemplares, como el umbral de calizas carboníferas situado a la altura de la entrada al valle de Cardaño de Arriba, en el talud de la carretera (Fig. 3), que presenta su

Figura 3

UMBRAL DE LAS CALIZAS DEL ESPIGÜETE A LA ENTRADA DEL VALLE DE CARDAÑO DE ARRIBA, A $1310 \mathrm{M}$, RECIENTEMENTE EXHUMADO POR LAS OBRAS DE LA CARRETERA Y QUE HAN DESTRUIDO SU ZONA INFERIOR. SE OBSERVA LA SUPERFICIE PULIDA Y ESTRIADA, ASI COMO MARCAS DE ARRANQUE Y DESALOJO DEBIDAS EL PASO DEL HIELO DEL GLACIAR. LAS ESTRÍAS SE HAN PRESERVADO DE LA DISOLUCIÓN AL ESTAR PROTEGIDAS POR EL TILL SUBGLACIAR, VISIBLE EN LA PARTE SUPERIOR DERECHA DE LA IMAGEN

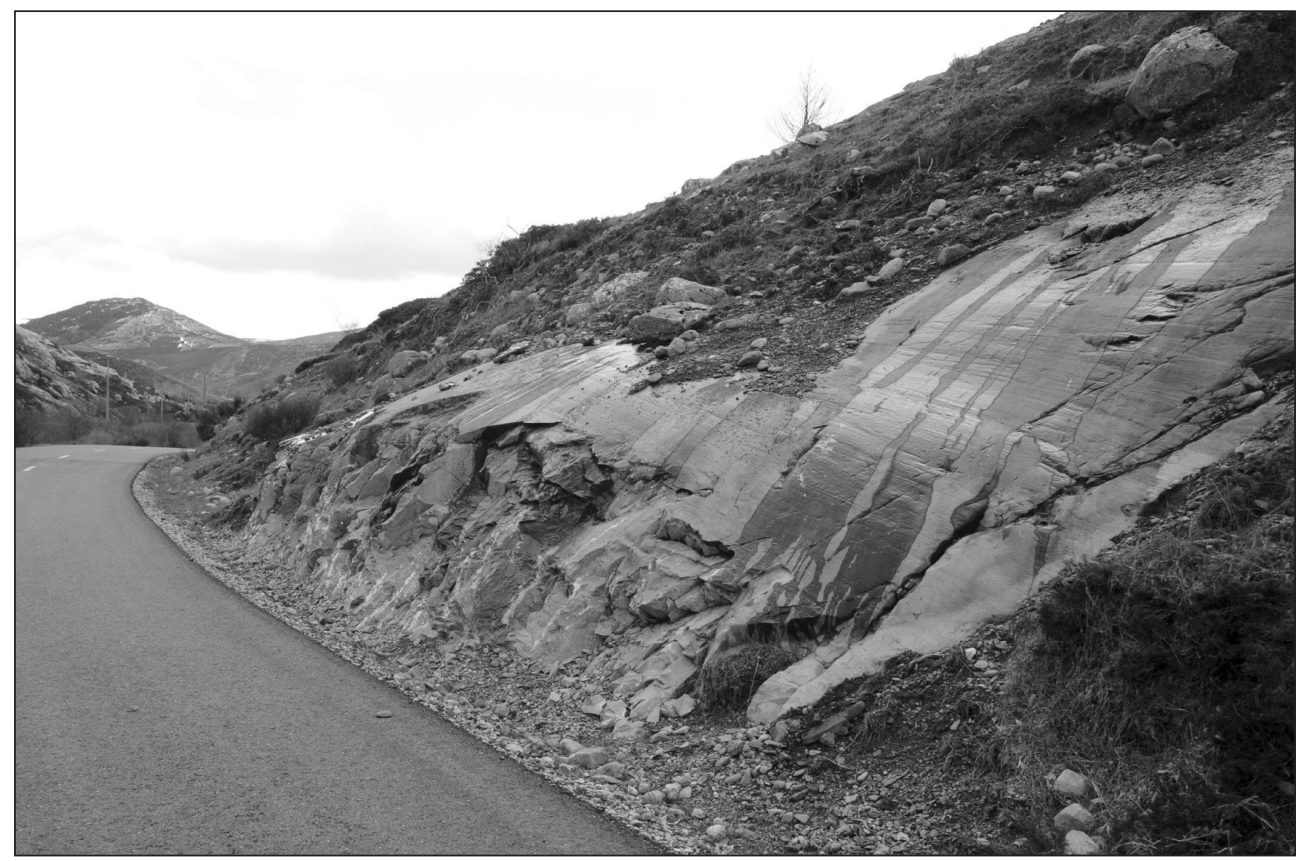


superficie perfectamente pulida, con marcas de arranque y desalojo por el hielo y estrías y acanaladuras unidireccionales. Su preservación a pesar de estar en calizas se debe a la labor de protección que ejercían los sedimentos glaciares, recientemente desprendidos debido a las obras realizadas en la carretera de acceso al pueblo.

\section{Los depósitos glaciares y las formas de acumulación}

Los depósitos de origen glaciar abundan por todo el valle; restos de till y bloques erráticos aparecen dispersos por muchos puntos. Además, la presencia aislada de granodioritas solo en el cordal del valle principal es un dato clave, pues permite la reconstrucción de la dinámica glaciar.

En la zona superior del valle se conservan algunas acumulaciones morrénicas laterales, como las localizadas a ambos lados, y por debajo, del mencionado circo de Hoya Continua, o las del valle del arroyo de La Lomba, aguas abajo de la laguna de Pozo Lomas (la lateral izquierda se ha cartografiado como «loma en acumulación morrénica», Frochoso Sánchez y Castañón Álvarez, 1996).

Gran interés tiene el valle de río Chico, que desciende de la vertiente meridional del Espigüete y desemboca en el río Cardaño en el entorno de Cardaño de Abajo. Las huellas morfológicas visibles indican que solo mantuvo pequeños glaciares de circo colgados en la pared sur del Espigüete, que tendrían muy corto recorrido, estando libre de hielo el resto de su cuenca. Por ello, el valle estuvo obturado por el glaciar de Cardaño, generándose un lago en su tramo final, como parecen indicar la existencia de limos y arcillas en la confluencia del Arroyo de la Binesa con el río Chico.

Gran singularidad tiene la presencia de numerosos bloques erráticos de granodiorita a lo largo de buena parte del valle principal. Los más destacados aparecen en la parte baja del valle, apoyados sobre las Calizas de Montaña (Rodríguez Fernández, 1984) (Fig. 4). En esta zona se encuentran numerosos bloques (algunos de entre 1 y $2 \mathrm{~m}$ de eje mayor) hasta 210 $\mathrm{m}$ por encima del fondo de valle actual, lo que permite inferir el espesor que tuvo el glaciar durante la fase de máximo avance. Los mismos bloques son visibles también en las calizas de la falda oriental del Espigüete, hasta $220 \mathrm{~m}$ sobre el río Cardaño, a la altura de la desembocadura el Arroyo Mazobres. Es destacable que por encima de los bloques erráticos, las calizas presentan un grado de disolución mucho mayor (con lapiaces bien desarrollados) que por debajo, dónde sólo aparecen formas débilmente evolucionadas.

La carretera que sigue todo el embalse, a ambos lados del valle de Cardaño de Abajo, corta en sus taludes y en varios puntos, restos de till glaciar en los que aparecen todas las rocas representativas de las estructuras situadas al $\mathrm{N}$, destacando la presencia de cantos aislados de granodiorita, grandes bloques de conglomerados y de cuarzoarenitas. Por otro lado, son relativamente abundantes los cantos estriados de arenisca. Así, en la margen izquierda del valle, puede observase un till que descansa sobre el zócalo de pizarras al tiempo que se apoya, empotrándose, contra un resalte de conglomerados. Este depósito contiene grandes bloques y cantos heterométricos y de distinta naturaleza (cuarcitas, calizas, areniscas, granodioritas), trabados con abundante matriz limo-arenosa y el conjunto conserva una elevada compacidad. Los bloques son subangulosos a subredondeados y a veces aparecen imbricados, con buzamiento al $\mathrm{N}$ y el eje mayor orientado en el sentido del valle (N-S); en general, 
Figura 4

BLOQUES ERRÁTICOS DE GRANODIORITA SOBRE LAS CALIZAS DE LA PARTE INFERIOR DEL VALLE DE CARDAÑO, ALA ALTURA DEL CRUCE DE CARDAÑO DE ARRIBA, A UNA ALTITUD DE $1350 \mathrm{M}$ (55 M SOBRE EL FONDO DE VALLEACTUAL)

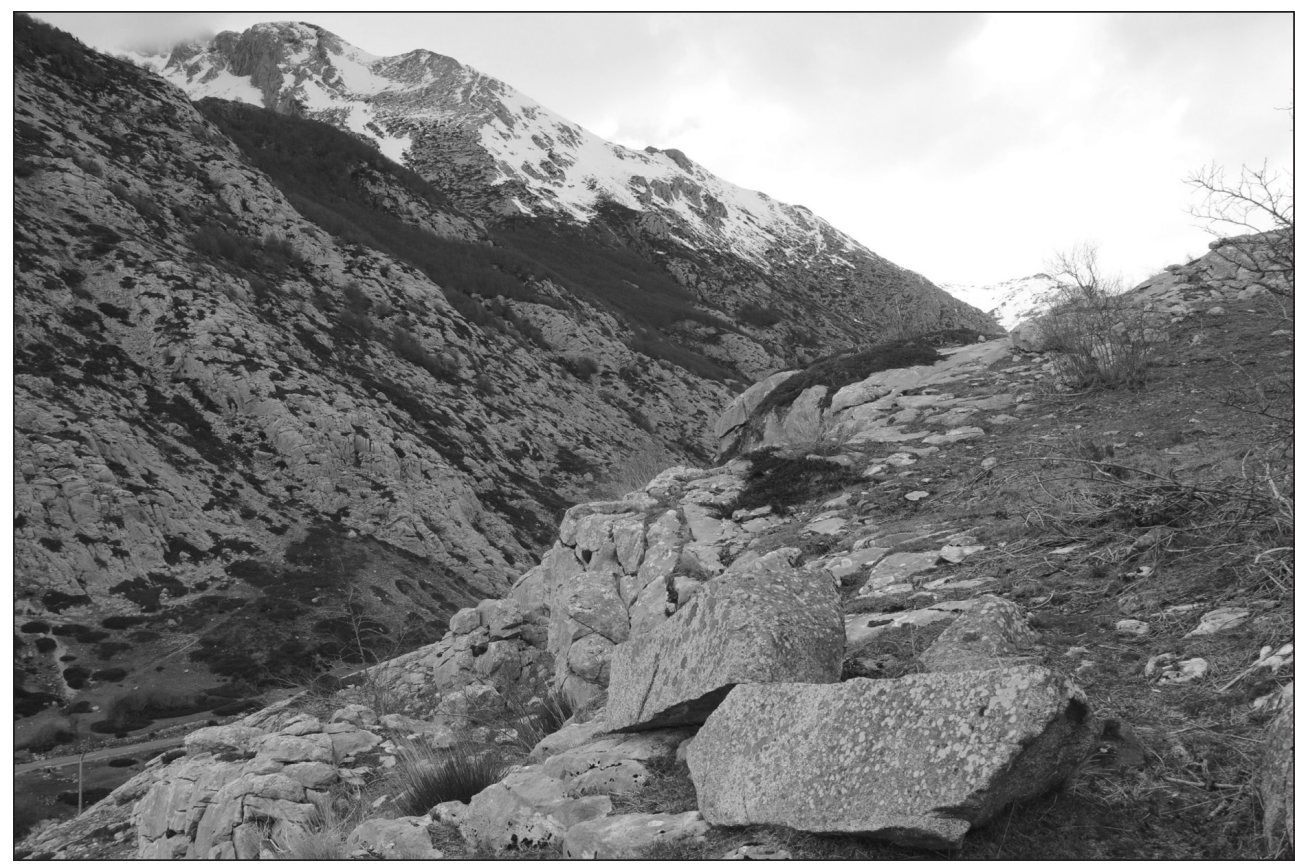

presentan un desgaste elevado, con las caras pulidas y las aristas embotadas (en algunos, sobre todo en las areniscas y las calizas, se observan estrías y marcas de arranque). Además, el depósito se encuentra fosilizado por otro de origen periglaciar formado por coladas solifluidales predominantemente pizarrosas.

Pero lo que realmente resulta singular de la herencia glaciar de este valle, es el complejo morrénico que se localiza en torno a lo que tuvo que ser el frente del glaciar, al menos durante la última glaciación. Aguas abajo de la localidad de Cardaño de Abajo, y estacionalmente sumergidas por las aguas del embalse, se localiza un conjunto de arcos morrénicos frontales (Fig. 5). Estas morrenas, ocultas bajo el agua gran parte del año (a excepción de la más interna de todas), emergen al final del verano a medida que se producen los desembalses. Así, van surgiendo sobre el nivel de las aguas los restos de las crestas morrénicas, quedando un tiempo aún sumergidas las depresiones intramorrénicas, que de otra forma pasarían casi inadvertidas dado el escaso desnivel entre estas y las crestas más externas.

Se conservan tres arcos morrénicos conectados con la margen derecha del valle, ya que por la izquierda el río los ha desmantelado prácticamente. El más externo es el situado a una cota inferior y presenta en realidad una doble cresta separada unos $100 \mathrm{~m}$ por un surco intermedio (Fig. 6: 1a y 1b). La topografía en torno a esta doble cresta es bastante caótica, con abundantes cantos y bloques estriados de areniscas y otros procedentes de las rocas de cabecera (conglomerados, cuarcitas, granodioritas). Las pequeñas irregularidades podrían 


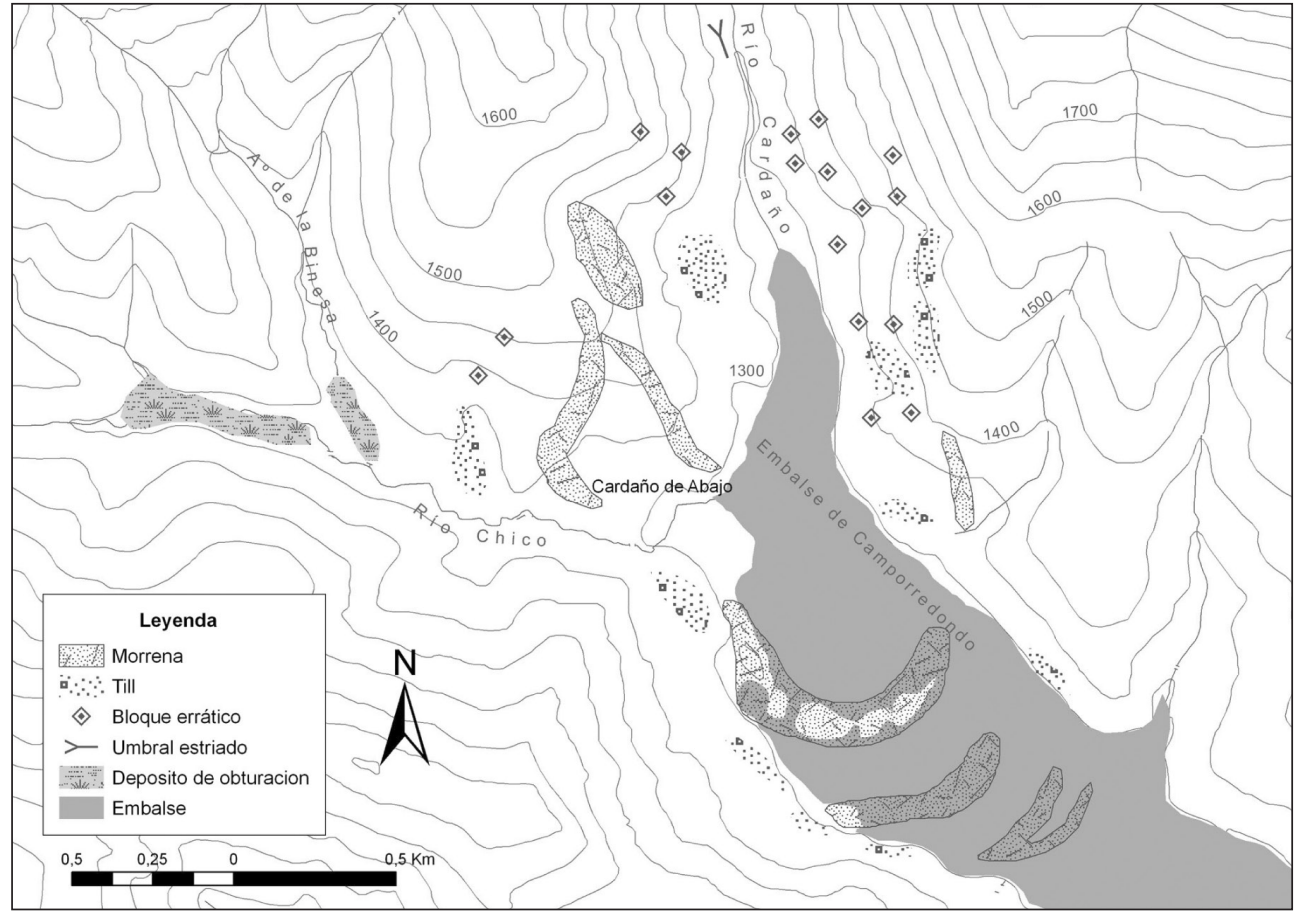

corresponder a las típicas de una zona próxima al frente de un glaciar, donde la desintegración del hielo, sobre todo ligada a un proceso de rápida retracción de este hacia posiciones más altas, genera ese tipo de superficies. No obstante, no se debe olvidar que todo este valle, antes de la construcción del embalse en 1930, tenía un intenso aprovechamiento agrario que tuvo que modificar también muchas de las formas de origen natural (aún son perceptibles las parcelas de cultivo con sus límites, la antigua carretera del valle, cercas de piedra). La cresta intermedia (Fig. 6: 2) tiene una mayor continuidad y está separada de las frontales por un surco de unos $250 \mathrm{~m}$ de longitud.

Por último, el arco interno (Fig. 6: 3) se sitúa en su parte central a una cota aproximada de 1292 m (es el mencionado cierre morrénico a 1250 m por varios autores: Nussbaum y Gigax, 1953; Frochoso Sánchez y Castañón Álvarez, 1996). Es el mejor conservado y desarrollado y está separado ya algo más de $300 \mathrm{~m}$ del intermedio. En su cresta conserva varios bloques erráticos (alguno de conglomerado de gran tamaño). La forma de media luna casi perfecta de este depósito es un signo de estabilización post-máximo prolongada de la lengua de hielo.

Las morrenas laterales correspondientes a estos arcos frontales se han conservado parcialmente y son visibles a la salida del río Cardaño de las Calizas de Montaña. Estas morrenas han sufrido una intensa erosión postglaciar y están afectadas por numerosos deslizamientos que dejan ver la estructura interna del depósito, caracterizado por la presencia de una matriz 


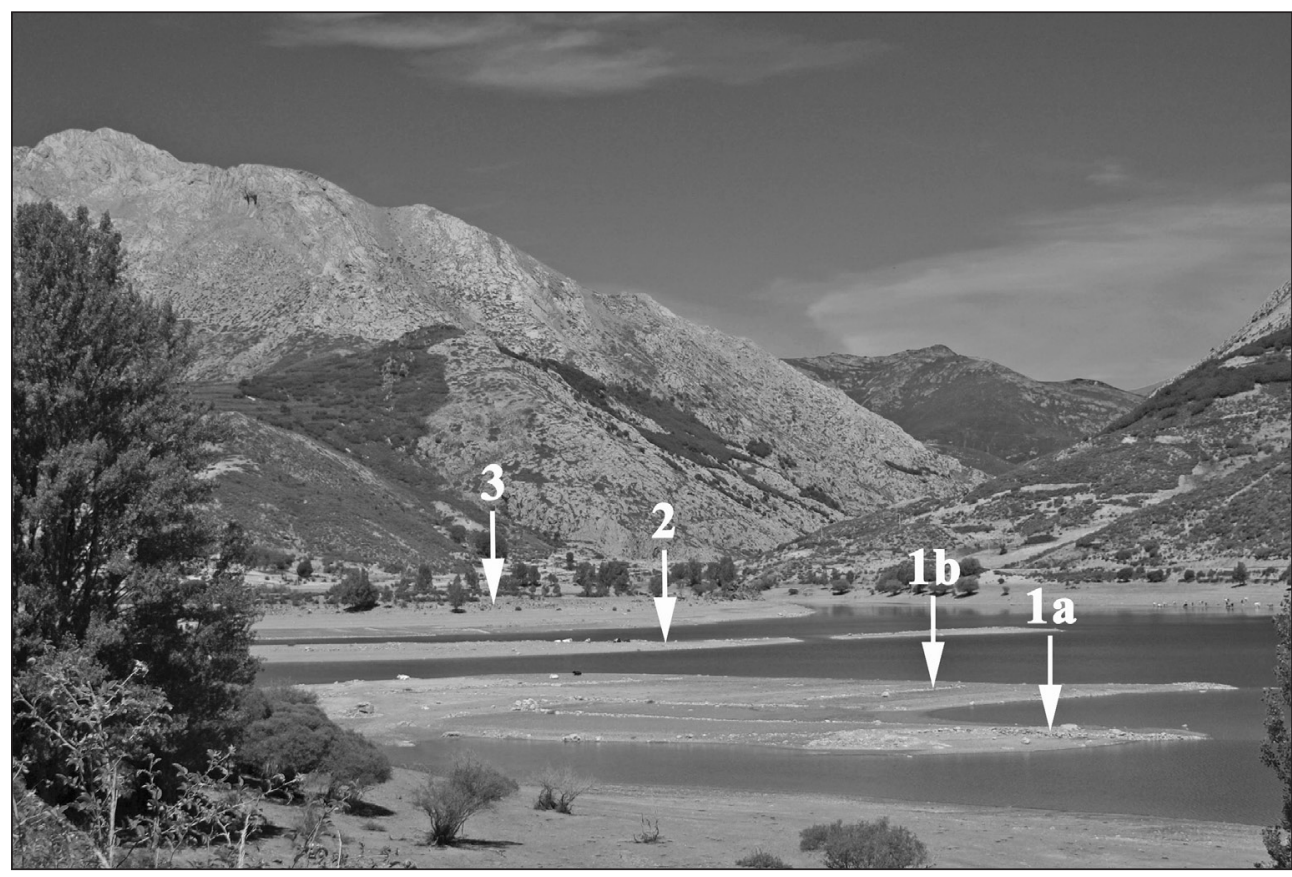

de materiales finos que envuelve clastos de litologías y tamaños muy variados, con presencia frecuente de estrías, sobre todo en las areniscas. Es destacable la gran pendiente media que presentan estas morrenas a partir de la confluencia del río Chico, indicando que el glaciar perdía espesor de forma muy rápida en esa zona al ampliarse el valle notablemente.

\section{LA ALTITUD DE EQUILIBRIO GLACIAR}

La gran cantidad de formas erosivas y de acumulación presentes en este valle, ha permitido reconstruir con un elevado nivel de detalle la dinámica glaciar, especialmente de la zona baja del valle, algo básico para poder estimar de forma lo más aproximada posible la altitud de la línea de equilibrio glaciar. Así, una vez definidos los límites del hielo en cada zona, se ha reconstruido la superficie ocupada por los glaciares y su hipsometría, que permite inferir el volumen de hielo que había por rangos altitudinales (Fig. 7, Tabla 1).

Aunque con la incertidumbre que supone la estimación de los espesores de hielo y de los límites exactos que alcanzó en algunas zonas, la gran cantidad de restos morfológicos y el uso de diversos valores de AABR, permiten determinar con bastante precisión la LEG durante la fase de máximo. Así, teniendo en cuenta la distribución del hielo por rangos altitudinales reseñada en la Tabla 1 y aplicando el método de cálculo de la LEG de Osmaston 


\section{Figura 7}

RECONSTRUCCIÓN DEL GLACIAR DE CARDAÑO Y SU HIPSOMETRÍA A PARTIR DE LOS RESTOS MORFOLÓGICOS. ISOHIPSAS CADA $100 \mathrm{M}$

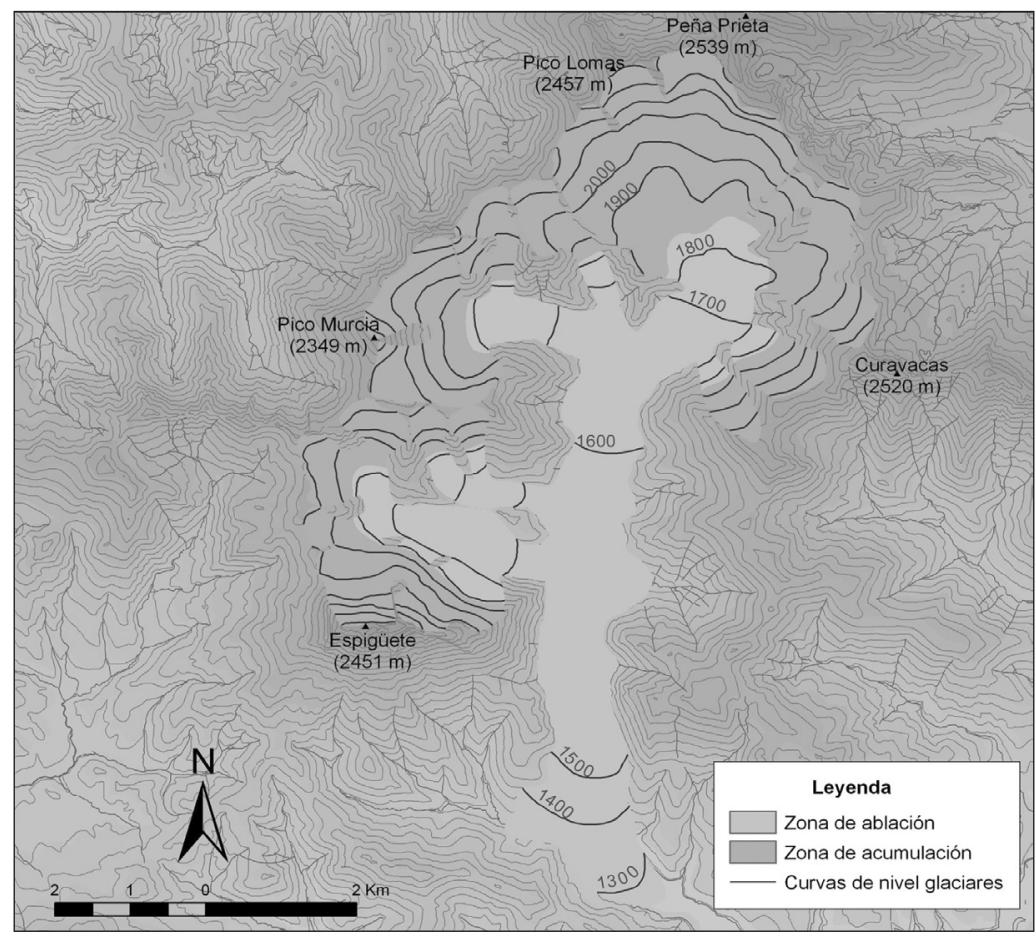

Tabla 1

SUPERFICIE GLACIAR POR RANGOS ALTITUDINALES DURANTE LA FASE DE MÁXIMO EN EL VALLE DEL CARDAÑO

\begin{tabular}{|c|c|}
\hline Altitud & Superficie \\
\hline $1260-1300$ & 23,40 \\
\hline $1300-1400$ & 125,19 \\
\hline $1400-1500$ & 83,36 \\
\hline $1500-1600$ & 492,69 \\
\hline $1600-1700$ & 447,09 \\
\hline $1700-1800$ & 309,76 \\
\hline $1800-1900$ & 418,44 \\
\hline $1900-2000$ & 442,17 \\
\hline $2000-2100$ & 470,23 \\
\hline $2100-2200$ & 287,82 \\
\hline $2200-2300$ & 108,39 \\
\hline $2300-2400$ & 61,26 \\
\hline $2400-2451$ & 3,34 \\
\hline Total & 3273,14 \\
\hline
\end{tabular}




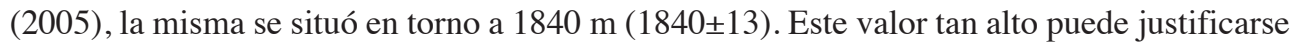
en parte por la orientación meridional de buena parte de la zona de acumulación, que haría que tuviera unas tasas de fusión muy elevadas, aunque otros valles de la zona con mejor orientación, como Lechada, Naranco o Carrión, debieron tener altitudes de la LEG similares, lo que más lleva a pensar a unas condiciones secas durante el máximo en el macizo de Peña Prieta.

Si bien aún no se han realizado reconstrucciones detalladas de la dinámica glaciar de muchos de los valles de la Cordillera, parece claro que la LEG tuvo altitudes fuertemente contrastadas entre unos sectores y otros siendo, en general, más baja cuanto más nos desplacemos a los extremos occidental y oriental y, también, hacia el extremo norte. Por ejemplo, en el macizo de Castro Valnera, Serrano y Gutiérrez Morillo (2002) sitúan la paleo-LEG entre 1100 y 1300 m. En otras sierras, como la de Aralar (Ugarte, 1992), la de Cabezo Lloroso (Castañón Álvarez, 1990), la Sierra del Palo (Alonso, 1998) o la del Caurel (Rodríguez Guitián et al., 1995) el valor debió ser muy similar. En la Sierra de Ancares se situó a 1350 m (Valcárcel Díaz y Pérez Alberti, 1998), al igual que en la cabecera del Ibias (Alonso, 1992).

En el otro extremo, los valores de paleo-LEG más elevados los sitúan Serrano y Gutiérrez Morillo (2002) para la zona de Alto Campoo (1700 m) y Valdecebollas (1800-1900 m), si bien en este último entorno aparecen restos glaciares dispersos que, de confirmarse, indicarían una LEG más baja durante el máximo (Fernández Martínez et al., 2009: nº 8). En la parte central de la Cordillera, González Gutiérrez (2002) establece la LEG entre 1500 m y 1700 m en la vertiente meridional, y Jiménez Sánchez (1996) a 1550 m en la septentrional, valor similar al señalado por Santos González (2012) en el Alto Sil.

Aunque en otras zonas pueden estimarse valores de la LEG para fases de retroceso, en el valle de Cardaño son muy escasos los restos deposicionales dejados después del máximo, por lo que no es posible realizar cálculos detallados de otras etapas. Únicamente debemos destacar las morrenas situadas en algunos de los circos por encima de los $2000 \mathrm{~m}$, cuando los glaciares se acantonaron en las zonas más elevadas. Posteriormente, aún se observan evidencias de una dinámica periglaciar que ha dejado algún ejemplo de glaciar rocoso, de tipo lobulado, como el situado en el Pico Murcia (Redondo Vega et al., 2010).

\section{CONCLUSIONES}

Durante el último máximo glaciar, la línea de equilibrio glaciar se situó en el valle de Cardaño a unos $1840 \mathrm{~m}$, valor relativamente elevado en el contexto de la Cordillera Cantábrica. Sin embargo, la configuración del relieve, con amplias superficies por encima de los 1800 m (e incluso de los $2000 \mathrm{~m}$ ) permitió el desarrollo de uno de las mayores lenguas glaciares de la Cordillera Cantábrica, con 13,1 km de desarrollo y algo más de 3200 ha de superficie.

Pero el mayor interés no radica en sus dimensiones, puesto que fue superado por otras lenguas como las del Sil, Trueba, Porma, Ibias, Valle Gordo o Carrión, sino en el elevado grado de conservación que presentan algunos de sus arcos morrénicos frontales, así como en la gran variedad de restos erosivos y sedimentarios que dejaron los glaciares, entre los que destacan los circos, las cubetas, los umbrales pulidos, los bloques erráticos y los valles de obturación. En conjunto, se puede afirmar que los restos glaciares del Valle de Cardaño constituyen un patrimonio natural de elevado interés científico y didáctico que debe ser conservado. 


\section{AGRADECIMIENTOS}

Este artículo tiene su origen en los hallazgos realizados por los autores durante la campaña de verano de 2008 del proyecto titulado «Estudio y catalogación de los Puntos de Interés Geológico y Paleontológico de las provincias de León y Palencia» financiado por la Consejería de Medio Ambiente de la Junta de Castilla y León. Agradecemos a los dos revisores anónimos las aportaciones realizadas al texto.

\section{BIBLIOGRAFÍA}

ALONSO, V. (1992): Geomorfología de las cabeceras de los ríos Narcea, Navia y Sil y del Parque Nacional de la Montaña de Covadonga (NO de la Península Ibérica). Tesis Doctoral. Oviedo. Dpto. de Geología, Universidad de Oviedo, 366 pp.

ALONSO, V. (1998): «El glaciarismo de la comarca de Laciana y alrededores. Zona occidental de la Cordillera Cantábrica» en Las huellas glaciares de las montañas españolas (Gómez Ortiz, A. y Pérez Alberti, A., eds.). Santiago de Compostela, Universidade de Santiago de Compostela, 139-160.

ALONSO, V. y SUÁREZ RODRÍGUEZ, A. (2004): «Evidencias geomorfológicas de la existencia de un pequeño casquete glaciar en la Comarca de Babia Alta (Cordillera Cantábrica)». Revista de la Sociedad Geológica de España, nº 17 (1-2), 61-70.

ALONSO HERRERO, E. (1987): «Huellas del glaciarismo cuaternario en las cabeceras del río Esla, Vertiente Sur de la Cordillera Cantábrica, (León)». Cuaternario y Geomorfología, $\mathrm{n}^{\circ} 1,49-59$.

ARENILLAS PARRA, M., SÁENZ RIDRUEJO, C., y ALONSO OTERO, F. (1982): «La morfología glaciar en las montañas de Castilla la Vieja y León» en El espacio geográfico de Castilla la Vieja y León (García Fernández, J., dir.). Burgos, 23-44.

BENN, D.I., y EVANS, D.J.A. (1998): Glaciers and Glaciation. London. Arnold.

BENN, D.I. y GEMMELL, A.M.D. (1997): «Calculating equilibrium-line altitudes of former glaciers by the balance ratio method: a new computer spreadsheet». Glacial Geology and Geomorphology. Disponible en http://ggg.qub.ac.uk/ggg/full/1997/tn011997/tn01.html

CASTAÑÓN ÁLVAREZ, J.C. (1989): Las formas de relieve de origen glaciar en los sectores central y oriental de Macizo Asturiano. Tesis Doctoral. Oviedo. Dpto. de Geografía, Universidad de Oviedo, 787 pp.

CASTAÑÓN ÁLVAREZ, J.C. (1990): «Datos para un estudio geomorfológico de la Sierra de Cabezo Lloroso (oriente de Asturias)». Ería, nº 23, 258-264.

FERNÁNDEZ MARTÍNEZ, E., FUERTES GUTIÉRREZ, I., ALONSO HERRERO, E., REDONDO VEGA, J.M., CORTIZO ÁLVAREZ, J., GÓMEZ VILLAR, A., SANTOS GONZÁLEZ, J., HERRERO HERNÁNDEZ, A. y GONZÁLEZ GUTIÉRREZ, R.B. (2009): Lugares de Interés Geológico. Palencia. DVD publicado por la Fundación Patrimonio Natural, Junta de Castilla y León.

FROCHOSO SÁNCHEZ, M. y CASTAÑÓN ÁLVAREZ, J.C. (1996): «El relieve heredado de la glaciación cuaternaria en el Macizo de Peña Prieta (Cordillera Cantábrica)». Polígonos, $\mathrm{n}^{\circ} 6,25-43$. 
FROCHOSO SÁNCHEZ, M. y CASTAÑÓN ÁLVAREZ, J.C. (1998): «El relieve glaciar de la Cordilllera Cantábrica» en Las huellas glaciares en las montañas españolas (Gómez Ortiz, A. y Pérez Alberti, A., eds.). Santiago de Compostela, Universidad de Santiago de Compostela, 65-137.

FURBISH, D.J. y ANDREWS, J.T. (1984): «The use of hypsometry to indicate long-term stability and response of valley glaciers to changes in mass transfer». Journal of Glacio$\log y, \mathrm{n}^{\mathrm{o}} 30$ (104), 199-211.

GARCÍA DE CELIS, A. y MARTÍNEZ FERNÁNDEZ, L.C. (2002): «Morfología glaciar de las montañas de la cuenca alta de los ríos Sil, Omaña, Luna y Bernesga: revisión y nuevos datos (Montaña Occidental de León)» en El modelado de origen glaciar en las montañas leonesas (Redondo Vega, J.M., Gómez Villar, A., González Gutiérrez, R.B. y Carrera Gómez, P., coords.). León, Universidad de León, 137-196.

GONZÁLEZ GUTIÉRREZ, R.B. (2002): El relieve de los valles del Torío y Curueño (Montaña Cantábrica Leonesa). León. Universidad de León.

GONZÁLEZ TRUEBA, J.J. (2006): El Macizo Central de los Picos de Europa: Geomorfología y sus implicaciones geoecológicas en la alta montaña cantábrica. Tesis doctoral. Santander. Dpto. de Geografía, Urbanismo y Ordenación del Territorio, Universidad de Cantabria, 819 pp.

GUTIÉRREZ ÁLVAREZ, R. (2007): «Valverde de la Sierra: la carretería de la nieve». Revista Comarcal Montaña de Riaño, $\mathrm{n}^{\circ} 22$.

HEREDIA CARBALLO, N. y RODRÍGUEZ FERNÁNDEZ, R. (2001): «Paisaje geológico de la provincia de Palencia» en Patrimonio geológico de Castilla y León (Nuche del Rivero, R., ed.). Madrid, Enresa, 192-223.

JALUT, G., TURU I MICHELS, V., DEDOUBAT, J.J., OTTO, T., EZQUERRA, J., FONTUGNE, M., BELET, J.M., BONNET, L., GARCÍA DE CELIS, A., REDONDO VEGA, J.M., VIDAL ROMANÍ, J.R. y SANTOS, L. (2010): «Palaeoenvironmental studies in NW Iberia (Cantabrian range): vegetation history and synthetic approach of the last deglaciation phases in the western Mediterranean». Palaeogeography, Palaeoclimatology, Palaeoecology, n 297 (2), 330-350.

JIMÉNEZ SÁNCHEZ, M. (1996): «El glaciarismo en la cuenca alta del río Nalón (NO de España): una propuesta de evolución de los sistemas glaciares cuaternarios en la Cordillera Cantábrica». Revista de la Sociedad Geológica de España, nº 9 (3-4), 157-168.

MEIER, M.F. y POST, A.S. (1962): Recent variations in mass net budges of glaciers in western North America. International Association of Hydrological Sciences, Publication 58: 63-77.

NUSSBAUM, F. y GIGAX, F. (1953): «La glaciación Cuaternaria en la Cordillera Cantábrica». Estudios Geográficos, n ${ }^{\circ}$ 51, 261-270.

OSMASTON, H. (2005): «Estimates of glacier equilibrium line altitudes by the Area $\mathrm{x}$ Altitude, the Area x Altitude Balance Ratio and the Area x Altitude Balance Index methods and their validation». Quaternary International, $\mathrm{n}^{\circ} 138-139,22-31$.

PÉREZ ALBERTI, A. y VALCÁRCEL DÍAZ, M. (1998): «Caracterización y distribución espacial del glaciarismo en el Noroeste de la Península Ibérica» en Las huellas glaciares de las montañas españolas (Gómez Ortiz, A. y Pérez Alberti, A., eds.). Santiago de Compostela, Universidade de Santiago de Compostela, 17-62. 
PRADO, C. DE (1852): «Notes sur les blocs erratiques de la Chaine Cantabrique». Bulletin Societè Geologique de France, T IX, 171-175.

REA, B.R. (2009): «Defining modern day Area-Altitude Balance Ratios (AABRs) and their use in glacier-climate reconstructions». Quaternary Science Reviews, no 28 (3-4), 237 248.

REDONDO VEGA, J.M. (2002): «El relieve glaciar de la Sierra de Gistredo (NW de la Cordillera Cantábrica, León)» en El modelado de origen glaciar en las montañas leonesas (Redondo Vega, J.M., Gómez Villar, A., González Gutiérrez, R.B. y Carrera Gómez, P., Coords.). León, Universidad de León, 105-136.

REDONDO VEGA, J.M., GÓMEZ VILLAR, A., GONZÁLEZ GUTIÉRREZ, R.B. y SANTOS GONZÁLEZ, J. (2010): Los glaciares rocosos de la Cordillera Cantábrica. León. Universidad de León.

RODRÍGUEZ FERNÁNDEZ, L.R., (Dir.) (1984): Mapa Geológico de España, E 1:50000, $n^{\circ} 106$ Camporredondo de Alba. Madrid. IGME.

RODRÍGUEZ FERNÁNDEZ, L.R., (Dir.) (1994): Mapa Geológico de España, E 1:50000, $n^{o} 81$ Potes. Madrid. ITGE

RODRÍGUEZ GUITIÁN, M.A., VALCÁRCEL DÍAZ, M. y PÉREZ ALBERTI, A. (1995): «Morfogénesis glaciar en la vertiente meridional de la Serra do Courel (NW Ibérico): El valle de A Seara» en Avances en la reconstrucción paleoambiental de las áreas de montaña lucenses (Pérez Alberti, A. y Martínez Cortizas, A., coords.). Lugo, Diputación Provincial de Lugo, 77-88.

SAINT-SAUD, A.A. (1893): «Les Picos de Europa (Monts Cantabriques). Etude Orographique». Annales Club Alpine Français, 38.

SANTOS GONZÁLEZ, J. (2012) : Glaciarismo y periglaciarismo en el Alto Sil, provincia de León (Cordillera Cantábrica). León. Universidad de León, 689 pp (CD-ROM).

SAVAGE, J.F. (1967): «Tectonic Analysis of Lechada and Curavacas Synclines, Yuso Basin, Leon, NW Spain». Leidse Geologische Mededelingen, $\mathrm{n}^{\circ}$ 39, 197-224.

SERRANO, E. y GUTIÉRREZ MORILLO, A. (2002): «El glaciarismo pleistoceno en la vertiente meridional de la Cordillera Cantábrica (Montaña de Palencia, Cantabria y Burgos)» en Geomorfología y Paisaje. Guía de excursiones. VII Reunión Nacional de Geomorfología (Serrano, E., ed.). Valladolid, Sociedad Española de Geomorfología y Dpto. de Geografía-Universidad de Valladolid, 91-161.

TORSNES, I., RYE, N. y NESJE, A. (1993): «Modern and little ice age equilibrium-line altitudes on outlet valley glaciers from Jostedalsbreen, western Norway: an evaluation of different approaches to their calculation». Arctic Alpine Research, $\mathrm{n}^{\circ} 25$ (2), 106-116.

UGARTE, F.M. (1992): «Glacial and periglacial morphogenesis in the Basque mountains» en The Late Quaternary in the Western Pyrenean Region (Cearreta, A. y Ugarte, F., eds.). Bilbao, Universidad del País Vasco, 235-250. 